\title{
Origin of the hysteresis of the current voltage characteristics of superconducting microbridges near the critical temperature
}

\author{
D. Yu. Vodolazov ${ }^{1, *}$ and F. M. Peeters ${ }^{2}$ \\ ${ }^{1}$ Institute for Physics of Microstructures, Russian Academy of Sciences, 603950, Nizhny Novgorod, GSP-105, Russia \\ ${ }^{2}$ Departement Fysica,Universiteit Antwerpen (CGB), Groenenborgerlaan 171, B-2020 Antwerpen, Belgium
}

(Received 30 May 2011; revised manuscript received 20 August 2011; published 14 September 2011)

\begin{abstract}
The current voltage (IV) characteristics of short [with length $L \lesssim \xi(T)]$ and long $[L \gg \xi(T)$ ] microbridges are theoretically investigated near the critical temperature of the superconductor. Calculations are made in the nonlocal (local) limit when the inelastic relaxation length due to electron-phonon interactions $L_{i n}=\left(D \tau_{i n}\right)^{1 / 2}$ is larger (smaller) than the temperature-dependent coherence length $\xi(T)\left(D\right.$ is the diffusion coefficient, $\tau_{i n}$ is the inelastic relaxation time of the quasiparticle distribution function). We find that, in both limits, the origin of the hysteresis in the IV characteristics is mainly connected with the large time scale over which the magnitude of the order parameter varies in comparison with the time-scale variation of the superconducting phase difference across the microbridge in the resistive state. In the nonlocal limit, the time-averaged heating and cooling of quasiparticles are found in different areas of the microbridge, which are driven, respectively, by oscillations of the order parameter and the electric field. We show that, by introducing an additional term in the time-dependent Ginzburg-Landau equation, it is possible to take into account the cooling effect in the local limit too.
\end{abstract}

DOI: 10.1103/PhysRevB.84.094511

PACS number(s): 74.25.Op, 74.20.De, 73.23.-b

\section{INTRODUCTION}

The hysteresis of the current voltage characteristics of narrow [with width $W \lesssim \xi(T)$ ] superconducting microbridges is a well-known phenomena (see, for example, Refs. 1 and 2). There are many works where the hysteresis is explained by either an increasing of the effective temperature of quasiparticles due to Joule heating (see, for example, Refs. 3-6) or due to a finite relaxation time of the magnitude of the order parameter. ${ }^{7-13}$ From a general point of view, both mechanisms are connected with the deviation of the quasiparticle distribution function from equilibrium in the resistive state. The usage of the concept of effective temperature assumes that the electron-electron inelastic relaxation time $\tau_{e-e}$ is much shorter than the electron-phonon inelastic relaxation time $\tau_{e-p h}$ (below we use the notation $\tau_{i n}=\tau_{e-p h}$ ), and in such a case, one may use the quasiequilibrium approach for the nonequilibrium quasiparticle distribution function and introduce a coordinate and time-dependent effective temperature for quasiparticles (see, for example, the recent review ${ }^{14}$ ). In this case, from the kinetic equations for the quasiparticle distribution function $f(\epsilon)$, one may derive the heat-conductance equation and the Poisson equation for the electrostatic potential. ${ }^{14}$ By using this system of equations, it is relatively easy to find the nonequilibrium response of the system (for example, see Refs. 3-6). Because Joule dissipation is proportional to $I^{2}$, it is obvious that, at relatively small currents, the heating of quasiparticles becomes weak and the superconductor returns to the superconducting state (this current is usually called the retrapping current $I_{r}$ ) and the IV characteristics are hysteretic.

However, in many cases, one has the opposite situation at $T \sim T_{c}$, i.e., $\tau_{e-p h}=\tau_{i n} \ll \tau_{e-e}$ or they are of the same order of magnitude (in aluminium and zinc, for example). Then, one is not allowed to introduce an effective temperature and one must solve the kinetic equations for $f(\epsilon)$. In the quasiclassical approach, they are the Boltzmann-type equations [see Refs. 14-18 or Eqs. (1) and (2) below] coupled with the equation for the order parameter [see Eq. (3) below]. The situation is simplified in the so-called local limit when the inelastic relaxation length $L_{i n}=\left(D \tau_{i n}\right)^{1 / 2}$ is smaller than the coherence length $\xi(T)$, and the dynamics of $|\Delta|$ and the response of the superconductor can be obtained from the generalized time-dependent Ginzburg-Landau equation, which contains $\tau_{i n}$ explicitly [see Refs. 17 and 18 or Eq. (6) below].

By using the time-dependent Ginzburg-Landau equations, it was shown that a finite relaxation time of the magnitude of the order parameter can be the reason for the hysteresis of the IV characteristics in superconducting microbridges. ${ }^{1,7}, 10-13$ Qualitatively, in this case, the mechanism of hysteresis can be described in the following way. The finite voltage drop over the microbridge is connected with the appearance of one or several (for relatively long microbridges) phase-slip centers. ${ }^{1,19,20}$ In the resistive state, after the phase-slip event (when the magnitude of the order parameter $|\Delta| \rightarrow 0$ in one point), the phase difference $\delta \phi$ across the phase-slip core decreases instantly by $2 \pi$ after which it starts to grow in accordance with the Josephson relation $\partial \delta \phi / \partial t \sim V$. The larger the applied current, the faster it changes in time $\tau_{\delta \phi} \sim 1 / V \simeq 1 / I_{n} \sim 1 / I$. But, the time variation of the magnitude of the order parameter $\tau_{|\Delta|}$ is governed by a different mechanism. It is known that $\tau_{|\Delta|}$ depends on the inelastic relaxation time $\tau_{\text {in }}$ of the quasiparticle distribution function and, for example, in the spatially uniform case, one has $\tau_{|\Delta|} \simeq \tau_{i n} /\left(1-T / T_{c}\right)^{1 / 2}$ near $T_{c} \cdot{ }^{15,20}$ For many superconductors at relatively low temperature below $T_{c}$, one may have $\tau_{|\Delta|} \gg \tau_{\delta \phi}$. As a result, after the phase-slip event, $|\Delta|$ increases much slower in time than $\delta \phi$ and, when $\delta \phi$ becomes sufficiently large (in order to make the superconducting state unstable again), the order parameter is still small [it is smaller than one could expect from the dependence of $|\Delta|(\delta \phi)$ in the stationary superconducting state]. For this reason, oscillations of $|\Delta|$ may occur at $I<I_{c}$ due to the fact that the order parameter is dynamically suppressed in the resistive (nonstationary) state, and the dynamical phase-slip 
process stops at $I=I_{r}$ when the period of oscillation $T_{|\Delta|}$ becomes larger than $\tau_{|\Delta|}{ }^{13}$

Unfortunately, even the generalized time-dependent Ginzburg-Landau equations have, in principle, a very restricted range of applicability [i.e., the temperature interval $\Delta T<$ $0.01 T_{c}$ near the critical temperature (see, for example, Table 1 in Ref. 18)]. In the nonlocal limit [when $L_{i n} \gg \xi(T)$ ] at $T \sim T_{c}$, the IV characteristics were theoretically studied for short $[L \lesssim \xi(T)]$ superconducting microbridges in Refs. 21 and 22. Those authors found a footlike structure in the IV characteristics and cooling of the quasiparticles at relatively large voltages. In our recent work, ${ }^{23}$ we argued that, in Refs. 21 and 22 too, rough assumptions were used and that the IV characteristics are hyperboliclike and hysteretic at relatively large $\tau_{i n}$. We should note that the hysteretic behavior of IV characteristics was also found theoretically for very short $L<\xi(T=0)$ superconducting bridges in Refs. 24 and 25 .

The aim of this paper is to study the origin of the hysteresis in the IV characteristics beyond the applicability region of the generalized time-dependent Ginzburg-Landau equations and to compare the results in the local and nonlocal limits. We show that, in the nonlocal limit, the hysteresis of the IV characteristics of both short and long superconducting microbridges is mainly connected with the long relaxation time of $|\Delta|$, which coincides with the results found in the local limit. We found new effects that appear in the nonlocal limit: time-averaged heating and cooling of quasiparticles in different areas of the sample, which affect the hysteresis quantitatively. By adding a new term in the time-dependent Ginzburg-Landau equations, we show that cooling effects can also be incorporated in the local model. We also find that, in short superconducting microbridges, both within the local and nonlocal limits, the voltage at fixed current is a nonmonotonous function of $\tau_{i n}$, which generalizes the result of Refs. 7, 10, and 26 where a decrease of $V\left(\tau_{i n}\right)$ was found for small $\tau_{i n}$ within the local limit.

The paper is organized as follows. In Sec. II, we present the theoretical model. In Sec. III, we discuss the current voltage characteristics of short and long microbridges in a temperature interval where the simple time-dependent Ginzburg-Landau equations are valid (local limit). In Sec. IV, we discuss the lower temperature regime where a solution of the kinetic equations for $f(\epsilon)$ and the modified time-dependent GinzburgLandau (TDGL) equations are needed (nonlocal limit), and we present our results both for short (Sec. IV A) and long (Sec. IV B) superconducting microbridges. In Sec. V, we discuss the applicability range of our results and propose a modification of the TDGL equation. In Sec. VI, we present our conclusions.

\section{MODEL}

To simulate the phase-slip process in a superconductor, we use the kinetic equations derived in Refs. $15-18$ for dirty superconductors near $T_{c}$ :

$$
\begin{aligned}
N_{1} \frac{\partial \delta f_{L}}{\partial t}= & D \nabla\left[\left(N_{1}^{2}-R_{2}^{2}\right) \nabla \delta f_{L}\right]+D \nabla\left(j_{\epsilon} f_{T}\right) \\
& -\frac{N_{1}}{\tau_{i n}} \delta f_{L}-R_{2} \frac{\partial f_{L}^{0}}{\partial \epsilon} \frac{\partial|\Delta|}{\partial t}
\end{aligned}
$$

$$
\begin{aligned}
\frac{\partial}{\partial t} N_{1}\left(f_{T}+e \varphi \frac{\partial f_{L}^{0}}{\partial \epsilon}\right)= & D \nabla\left[\left(N_{1}^{2}+N_{2}^{2}\right) \nabla f_{T}\right]+D \nabla\left(j_{\epsilon} \delta f_{L}\right) \\
& -\frac{N_{1}}{\tau_{i n}}\left(f_{T}+e \varphi \frac{\partial f_{L}^{0}}{\partial \epsilon}\right)-N_{2}|\Delta| \\
& \times\left(2 f_{T}+\hbar \frac{\partial f_{L}^{0}}{\partial \epsilon} \frac{\partial \phi}{\partial t}\right) . \quad(1 \mathrm{~b})
\end{aligned}
$$

Here, $Q=(\partial \phi / \partial x-2 e A / \hbar c)$ is a quantity that is proportional to the superfluid velocity $\left(v_{s}=D Q\right), \varphi$ is the electrostatic potential, $f_{L}(\epsilon)=f_{L}^{0}(\epsilon)+\delta f_{L}(\epsilon)$ is the longitudinal and $f_{T}(\epsilon)$ is the transverse part of the quasiparticle distribution function $2 f(\epsilon)=1-f_{L}(\epsilon)-f_{T}(\epsilon)$ [in equilibrium, $\left.f_{L}=f_{L}^{0}(\epsilon)=\tanh \left(\epsilon / 2 k_{B} T\right), f_{T}^{0}(\epsilon)=0\right] . N_{1}, N_{2}, R_{2}$ are the spectral functions, which should be found from the Usadel equation for the normal $\alpha(\epsilon)=\cos \Theta=N_{1}(\epsilon)+i R_{1}(\epsilon)$ and anomalous $\beta_{1}=\beta e^{i \phi}, \beta_{2}=\beta e^{-i \phi}\left[\beta(\epsilon)=\sin \Theta=N_{2}(\epsilon)+\right.$ $i R_{2}(\epsilon)$ ] Green's functions

$$
\begin{aligned}
& \hbar D \frac{d^{2} \Theta}{d x^{2}}+\left[\left(2 i \epsilon-\frac{\hbar}{\tau_{i n}}\right)-\hbar D Q^{2} \cos \Theta\right] \sin \Theta \\
& +2|\Delta| \cos \Theta=0 .
\end{aligned}
$$

Equations (1a) and (1b) are coupled due to the finite spectral supercurrent $^{27} j_{\epsilon}=\operatorname{Re}\left(\beta_{1} \nabla \beta_{2}-\beta_{2} \nabla \beta_{1}\right) / 2=2 N_{2} R_{2} Q$.

Equations (1) and (2) should be completed by the equation for the complex order parameter $\Delta=|\Delta| e^{i \phi}$ :

$$
\begin{aligned}
& \frac{\pi \hbar}{8 k_{B} T_{c}} \frac{\partial \Delta}{\partial t}-\left(\Phi_{1}+i \Phi_{2}\right) \Delta \\
& =\xi_{\mathrm{GL}}^{2} \frac{\partial^{2} \Delta}{\partial x^{2}}+\left(1-\frac{T}{T_{c}}-\frac{|\Delta|^{2}}{\Delta_{\mathrm{GL}}^{2}}\right) \Delta,
\end{aligned}
$$

where $\xi_{\mathrm{GL}}^{2}=\pi \hbar D / 8 k_{B} T_{c}$ and $\Delta_{\mathrm{GL}}^{2}=8 \pi^{2}\left(k_{B} T_{c}\right)^{2} / 7 \zeta(3)$ are the zero-temperature Ginzburg-Landau coherence length and the order parameter correspondingly. Nonequilibrium corrections to the quasiparticle distribution function enters Eq. (3) via the potentials $\Phi_{1}=\int_{0}^{\infty} R_{2} \delta f_{L} d \epsilon /|\Delta|$ and $\Phi_{2}=$ $\int_{0}^{\infty} N_{2} f_{T} d \epsilon /|\Delta|$.

The current and electrostatic potential in the wire can be found using the equations

$$
\begin{gathered}
j=\frac{\sigma_{n}}{e}\left(\frac{|\Delta|^{2} Q}{4 k_{B} T_{c}}+\int_{0}^{\infty}\left[\left(N_{1}^{2}+N_{2}^{2}\right) \nabla f_{T}+j_{\epsilon} \delta f_{L}\right] d \epsilon\right) \\
e \varphi=-\int_{0}^{\infty} N_{1} f_{T} d \epsilon / \int_{0}^{\infty} N_{1} \frac{\partial f_{L}^{0}}{\partial \epsilon} d \epsilon
\end{gathered}
$$

where $\sigma_{n}$ is the conductivity of the normal state and it is assumed that the charge density $\rho \simeq 0$ in metals and that the condition $d i v j=0$ is satisfied due to Eqs. (1b), (3), and (5).

In the so-called local limit [when $L_{\text {in }} \ll \xi(T)$ and $\tau_{\text {in }} \ll$ $\tau_{\mathrm{GL}}$ ], one may obtain from Eqs. (1)-(4) (see Refs. 17 and 18) the much simpler time-dependent Ginzburg-Landau equation for the dynamics of the order parameter:

$$
\begin{aligned}
& \frac{\pi \hbar}{8 k_{B} T_{c}} \frac{1}{\sqrt{1+(\Gamma|\Delta|)^{2}}}\left(\frac{\partial}{\partial t}-\frac{i 2 e \varphi}{\hbar}+\frac{\Gamma^{2}}{2} \frac{\partial|\Delta|^{2}}{\partial t}\right) \Delta \\
& =\xi_{\mathrm{GL}}^{2} \frac{\partial^{2} \Delta}{\partial x^{2}}+\left(1-\frac{T}{T_{c}}-\frac{|\Delta|^{2}}{\Delta_{\mathrm{GL}}^{2}}\right) \Delta
\end{aligned}
$$




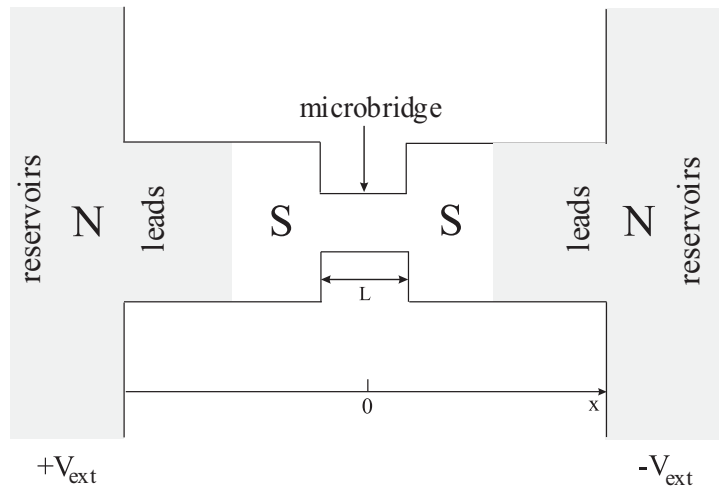

FIG. 1. Schematic illustration of the model system.

$\left(\Gamma=2 \tau_{\text {in }} / \hbar\right)$, and the current density

$$
j=\sigma_{n}\left(\frac{|\Delta|^{2} Q}{4 e k_{B} T_{c}}-\frac{\partial \varphi}{\partial x}\right)
$$

but which has a much narrower validity region (1$\pi \hbar / 8 k_{B} T_{c} \tau_{\text {in }} \lesssim T / T_{c}<1$ ) than Eqs. (1)-(5) (which roughly coincides with the validity region of the stationary GinzburgLandau equations: $0.9 \lesssim T / T_{c}<1$ ).

In our theoretical model, we assume that the quasi-onedimensional microbridge of finite length $L$ is connected to large superconducting leads (see Fig. 1), where the order parameter reaches the equilibrium value $\Delta_{\text {lead }} \simeq 1.74 \Delta_{0}(1-$ $\left.T / T_{s}\right)^{1 / 2}\left(\Delta_{0}=1.76 k_{B} T_{c}\right.$ is the zero-temperature order parameter value in the weak-coupling limit) and the current density is much smaller than in the microbridge. It is useful to introduce the dimensionless inelastic relaxation time $\tilde{\tau}_{i n}=$ $\tau_{i n} / t_{0}$, which is the main control parameter in the model described by Eqs. (1)-(3) [here, $t_{0}=\hbar / \Delta_{0}$ and, further, we also use $\xi_{0}=\sqrt{\hbar D / \Delta_{0}}, Q_{0}=\hbar c / 2 e \xi_{0}, j_{0}=\Delta_{0} \sigma_{n} /\left(\xi_{0} e\right)$, and $\varphi_{0}=\Delta_{0} / e$ as the units of the corresponding quantities). In our calculations, we used $\tilde{\tau}_{\text {in }}=1-1000$, which are typical values for many low-temperature superconductors (for example, in $\mathrm{Nb} \tilde{\tau}_{i n} \simeq 100$, in Sn $\tilde{\tau}_{i n} \simeq 200$, and in $\left.A l \tilde{\tau}_{\text {in }} \simeq 1000\right) .{ }^{18}$

To simulate the current driven regime in the nonlocal limit as described by Eqs. (1)-(5), we apply the special system present in Fig. 1. Usually, the boundary conditions for $f_{L}$ and $f_{T}$ contain the voltage as a parameter ${ }^{27,28}$ and it is rather difficult to satisfy the condition $j=$ const. Therefore, we apply a voltage to the system, which consists of a large normal part (reservoirs + part of the leads) and a superconducting part (microbridge + rest of the leads), which is joint in series and this system effectively models the regime of applied current. Details of the numerical procedure and the model can be found in Ref. 23.

In the local limit, the problem of the current-carrying state is much simpler because the full current is $j=j_{s}+j_{n}$, and this relation can be directly integrated using Eq. (7) to find the distribution of the electrostatic potential $\varphi$ with boundary conditions $\left.\varphi\right|_{-L / 2}=0,\left.\varphi\right|_{L / 2}=V$, where $V=\int_{-L / 2}^{L / 2}\left(j_{S}-\right.$ $j) d x / \sigma_{n}$. One then also can solve Eq. (6) in the interval $-L / 2<x<L / 2$ with boundary conditions $\left.\Delta\right|_{-L / 2}=\Delta_{\text {lead }}$, $\left.\Delta\right|_{L / 2}=\Delta_{\text {lead }} e^{i V t}\left[\right.$ where $\Delta_{\text {lead }}=\Delta_{\mathrm{GL}}\left(1-T / T_{c}\right)^{1 / 2}$ ] to find $\Delta(x, t), j_{s}(x, t), \varphi(x, t)$ and the time-averaged voltage response.
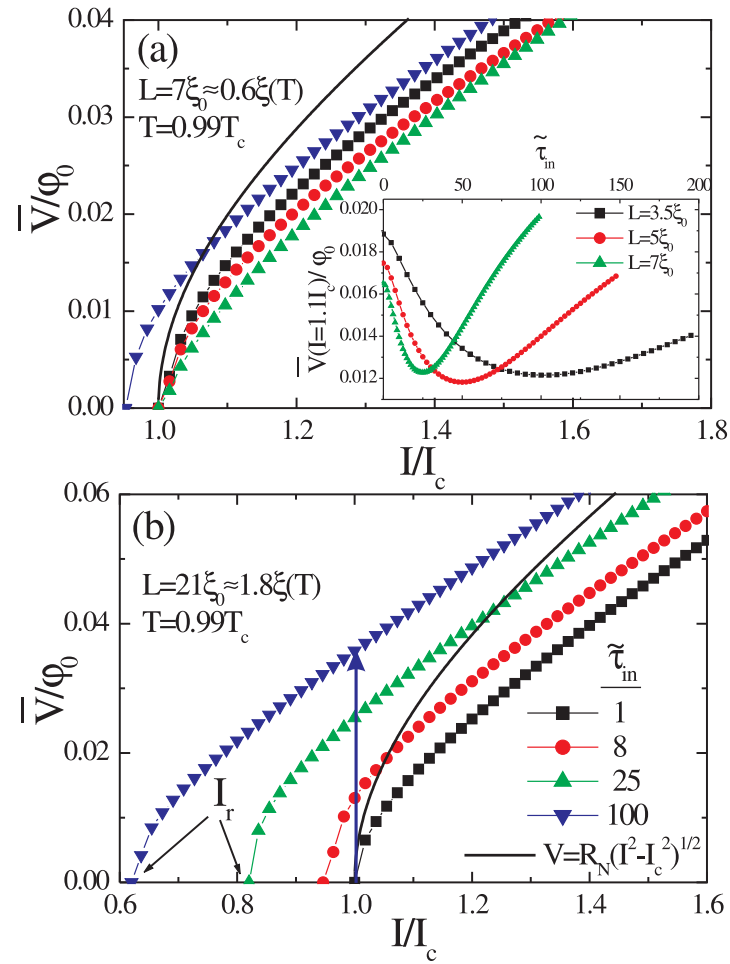

FIG. 2. (Color online) Current voltage characteristics of short (a) and relatively long (b) superconducting microbridges calculated in the regime of decreasing current using time-dependent GinzburgLandau equations. For comparison, we also plot the IV characteristics of the overdamped Josephson junction within the RCSJ model (solid curve).

\section{SHORT AND LONG MICROBRIDGES IN LOCAL LIMIT (TDGL FORMALISM)}

First, we consider the dynamical properties of short and long superconducting microbridges in the temperature interval where the time-dependent Ginzburg-Landau equations [Eqs. (6) and (7)] are valid.

In Fig. 2, we present typical current voltage characteristics of microbridges of different lengths calculated at $T=0.99 T_{c}$ in the regime of decreasing current for different values of $\tilde{\tau}_{i n}$. All of them have a hyperbolic shape and some of them are hysteretic (in the sense that the transition to the superconducting state occurs at the retrapping current $I_{r}<I_{c}$ ). For short microbridges, the voltage at fixed current $I \gtrsim I_{c}$ is a nonmonotonous function of $\tilde{\tau}_{i n}$ : it has a minimum at some $\tilde{\tau}_{i n}^{*}$, which is inversely proportional to $L^{2}$ [see inset in Fig. 2(a)]. For relatively long microbridges [with $L \gtrsim \xi(T)$ ], the voltage at fixed current monotonically increases with increase of $\tilde{\tau}_{i n}$ and the IV characteristics are hysteretic already for relatively small values of $\tilde{\tau}_{i n}$.

\section{A. Short microbridge}

Let us first discuss our results for short microbridges. We explain the nonmonotonous dependence $V\left(\tau_{i n}\right)$ and the hysteresis of the IV characteristics by the competition between the variation in time of $|\Delta|$ and $\delta \phi$. We may estimate these times using Eqs. (6) and (7). Because the electric field is almost uniform over the short microbridge, we may find from 
the Josephson relation $\partial \delta \phi / \partial t=2 e V / \hbar$ the growth time of $\delta \phi$ just after the phase-slip event: $\tau_{\delta \phi} \sim \pi \hbar / 4 e V \sim \hbar / R_{N} I \sim$ $\hbar I_{c} / I k_{B}\left(T_{c}-T\right)$ (where we use the expression for the critical current of a short microbridge $I_{c}=\pi \Delta_{\text {lead }}^{2} / 4 e k_{B} T_{c} R_{N}$ and assume that $\delta \phi$ increases by $\pi / 2$ ). In some respect, this time is proportional to the period of oscillations of $|\Delta|\left(T_{|\Delta|}\right)$ or the Josephson period. It is also related to the relaxation time of the current $\tau_{j}=\hbar / 2 e I_{c} R_{N}$ (see, for example, Ref. 1) in the superconductor: $\tau_{\delta \phi} \sim \tau_{j} I_{c} / I$.

To estimate $\tau_{|\Delta|}$, we find from Eq. (6) [by neglecting linear and nonlinear terms in the right-hand side of Eq. (6)]

$$
\frac{\partial|\Delta|^{2}}{\partial t} \sim \frac{\xi_{\mathrm{GL}}^{2} \Delta_{\text {lead }} k_{B} T_{c}}{L^{2} \tau_{i n}},
$$

and $\tau_{|\Delta|} \sim\left(L / \xi_{\mathrm{GL}}\right)^{2} \tau_{\text {in }}\left(1-T / T_{c}\right)^{1 / 2}$ [it is the time needed that $|\Delta|$ increases from zero to $\Delta_{\text {lead }}$ according to Eq. (8)]. The above estimates for $\tau_{\delta \phi}$ and $\tau_{|\Delta|}$ are very rough, but they allow us to understand qualitatively our numerical results in a simple way by introducing the ratio

$$
\frac{\tau_{|\Delta|}}{\tau_{\delta \phi}} \simeq \tilde{\tau}_{i n}\left(1-T / T_{c}\right)^{1 / 2} \frac{I}{I_{c}}\left(\frac{L}{\xi(T)}\right)^{2}, \quad L<\xi(T) .
$$

When $\tau_{\text {in }}$ is sufficiently small for given length of the microbridge and given temperature to make $\tau_{|\Delta|} / \tau_{\delta \phi} \ll 1$, the current-phase relation $I_{S}[(\delta \phi)]$ is close to the sinusoidal one [see Fig. 3(b)], and the dependence $|\Delta|(\delta \phi)$ in the resistive state is almost the same as in the superconducting state [see

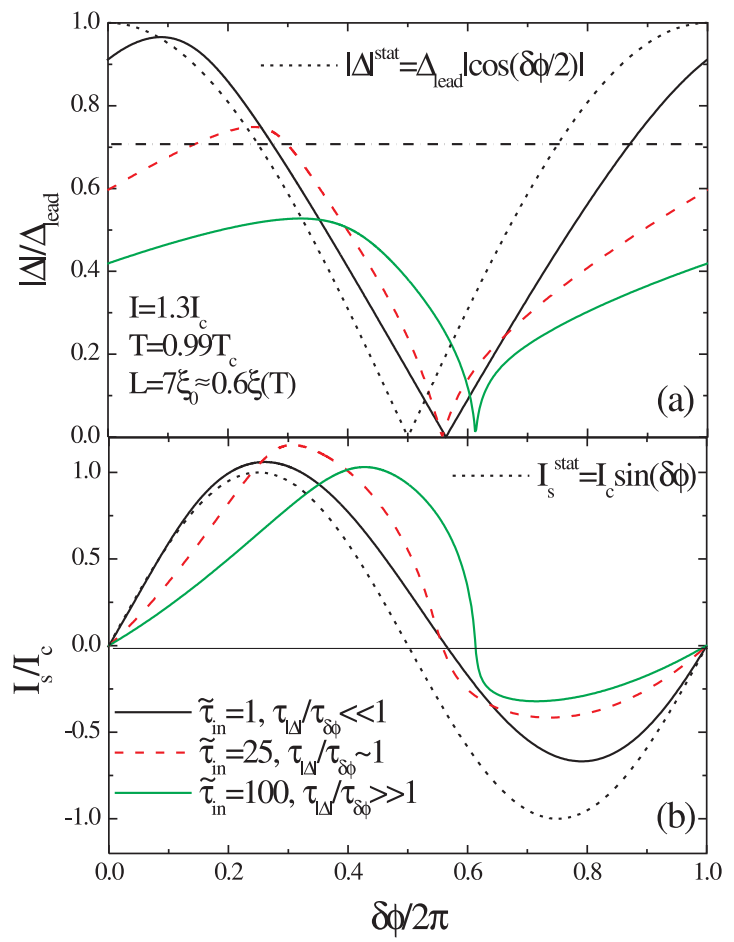

FIG. 3. (Color online) Dependence of the order parameter (a) and the superconducting current (b) in the center of the short microbridge $\left[L=7 \xi_{0} \sim 0.6 \xi(T)\right.$ at $\left.T=0.99 T_{c}\right]$ on the phase difference across the sample at $I=1.3 I_{c}$ and different inelastic relaxation times $\tilde{\tau}_{i n}$ found from the numerical solution of Eqs. (6) and (7). The horizontal dashed-dotted line in (a) shows the minimal value of $|\Delta|=\Delta_{\text {lead }} / \sqrt{2}$ in the stationary state when $\delta \phi=\pi / 2$ (Ref. 29).

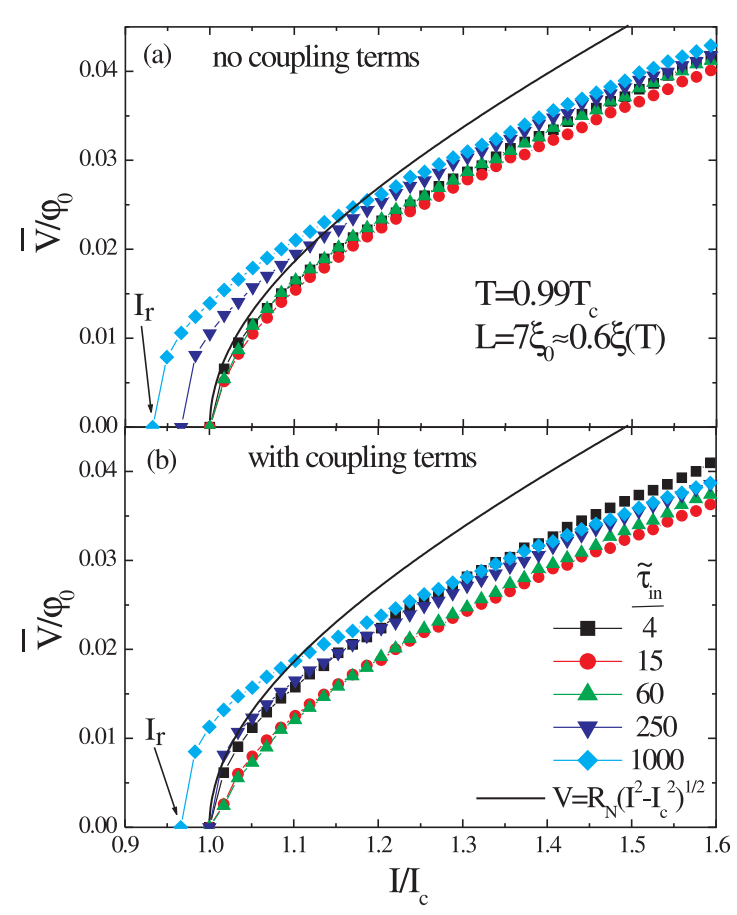

FIG. 4. (Color online) Current voltage characteristics (in the regime of decreasing current) of a short microbridge with length $L=7 \xi_{0}$ at $T=0.99 T_{c}$ calculated for different values of the inelastic relaxation time in the absence (a) and the presence (b) of coupling terms in Eqs. (1a) and (1b).

Fig. 3(a)]. In this case, the magnitude of the order parameter follows the changes in $\delta \phi$ without almost any delay.

When we increase $\tau_{\text {in }}$ (keeping the other parameters constant), $\tau_{|\Delta|}$ increases, but as long as $\tau_{|\Delta|} / \tau_{\delta \phi} \lesssim 1$, it leads to a decrease of the voltage at fixed current $I \gtrsim I_{c}$ because the variation time of $|\Delta|$ increases (it scales with $\tau_{i n}$ ) and, thus, more time is needed to change the order parameter from its minimal to its maximal value; consequently, the period $T_{|\Delta|}$ increases. In some respect, it resembles the response of the superconductor on the current pulse: ${ }^{30-32}$ the larger $\tau_{i n}$, the larger the decay time of $|\Delta|$. The increased $\tau_{|\Delta|}$ leads to a smaller maximal value $|\Delta|_{\max }$ during the variation of $\delta \phi$ and larger deviations of both dependencies $|\Delta|(\delta \phi)$ and $I_{S}(\delta \phi)$ from the one in the static case [see Figs. 3(a) and 3(b)].

With a further increase of $\tau_{i n}$, the amplitude of oscillations of $|\Delta|$ further decreases [compare curves in Fig. 3(a)] and there is a moment when the period of oscillations starts to decrease (and voltage $V \sim 1 / T_{|\Delta|}$ increases) despite the increase of $\tau_{|\Delta|}$. It occurs roughly when $\tau_{|\Delta|} / \tau_{\delta \phi} \gtrsim 1$ [see inset in Fig. 2(a)]. Approximately at the same values of $\tau_{i n}$, the IV characteristics become hysteretic. At such a $\tau_{i n}$, we can have a resistive state for $I<I_{c}$ because $|\Delta|$ increases very slowly after the phaseslip event (in comparison with $\delta \phi$ ), and when $\delta \phi$ approaches $\pi / 2$ (critical value for the superconducting microbridge in the static case), the magnitude of the order parameter is still strongly suppressed [see Fig. 3(a)]. Therefore, oscillations of $|\Delta|$ may still be present at the current $I \simeq I_{s} \sim|\Delta|_{\max }^{2} \pi / 2 L$, which can be smaller than $I_{c} \sim \Delta_{\text {lead }}^{2} \pi / 4 L$. We may estimate the retrapping current $I_{r}$, at which the dynamical phase-slip 
process stops, from the condition $\tau_{|\Delta|} / \tau_{\delta \varphi}\left(I_{r}\right) \lesssim 1$, which leads to

$$
I_{r} \lesssim \frac{I_{c}}{\tilde{\tau}_{i n}\left(1-T / T_{c}\right)^{1 / 2}}\left(\frac{\xi(T)}{L}\right)^{2}, \quad L<\xi(T) .
$$

At this current, the period of oscillations is about $\sim \tau_{|\Delta|}$ and, therefore, there is a minimal voltage $V^{*} \sim 1 / \tau_{|\Delta|}$ below which the resistive state is absent in the current-driven regime.

We have to stress that the above estimates are very rough and show only the trends as to how $I_{r}$ and $V^{*}$ change with the variation of length of the microbridge, the inelastic relaxation time, and temperature. The exact dependencies should be obtained from a numerical solution of Eqs. (6) and (7) (in the temperature interval where they are valid).

We may compare the dynamical properties of the short microbridge as obtained within the framework of the TDGL model [Eqs. (6) and (7)] with those of a Josephson junction within the framework of the resistively and capacitively shunted junction (RCSJ) model. ${ }^{20}$ The qualitative important difference with the RCSJ model is that, for a superconducting microbridge with a large ratio $\tau_{|\Delta|} / \tau_{\delta \phi}$, the current-phase relation is not sinusoidal in the resistive state [see Fig. 3(b)]. Let us use the analogy of the Josephson junction with an effective particle, which moves in the external potential $U_{\text {eff }}(\delta \phi)$ such as $-\partial U_{\text {eff }} / \partial \delta \phi=I_{s}(\delta \phi) .{ }^{20}$ In the case of the superconducting microbridge, the moving particle affects the potential in which it moves, and, the larger the ratio $\tau_{|\Delta|} / \tau_{\delta \phi}$, the stronger the effective potential $U_{\text {eff }}$ is modified. This is the main reason for the existence of the order parameter oscillations at $I<I_{c}$ because the effective potential $U_{\text {eff }}$ in the resistive state is different from the one in the static state.

We should note that the TDGL equations were already used to study the dynamical properties of short superconducting microbridges (see, for example, the review in Ref. 12). One usually introduced a numerical coefficient $u^{*}$ in front of the time derivative $\partial \Delta / \partial t$ [plus one should set $\tau_{i n}=0$ in Eq. (6)], which leads to

$$
\begin{aligned}
u^{*} \frac{\pi \hbar}{8 k_{B} T_{c}}\left(\frac{\partial}{\partial t}-\frac{i 2 e \varphi}{\hbar}\right) \Delta= & \xi_{\mathrm{GL}}^{2} \frac{\partial^{2} \Delta}{\partial x^{2}} \\
& +\left(1-\frac{T}{T_{c}}-\frac{|\Delta|^{2}}{\Delta_{\mathrm{GL}}^{2}}\right) \Delta,
\end{aligned}
$$

and one studies then the dynamics of the order parameter for different values of $u^{*}$. This parameter describes superconductors with different $\tau_{i n}$, i.e., with the replacement $u^{*} \rightarrow 2 \tau_{i n}|\Delta| / \hbar \gg 1$, one obtains Eq. (6) for dynamics of $|\Delta|$ from Eq. (11). It is easy to see that, in the framework of Eq. (11), we have for short microbridges $\tau_{|\Delta|} \sim u^{*}\left(L / \xi_{\mathrm{GL}}\right)^{2}$, and $\tau_{\delta \phi}$ is the same as in Eq. (6). In Ref. 7, one introduced the parameter $v=u^{*}[L / \xi(T)]^{2} I / I_{c} \sim \tau_{|\Delta|} / \tau_{\delta \phi}$ [see Eq. (22) in Ref. 7 or Eq. (168) in Ref. 1], which describes the influence of finite $\tau_{|\Delta|}$ on the IV characteristics and on the deviation from the stationary current-phase relation $I_{s}=$ $I_{c} \sin (\delta \phi)$. In this model, the IV characteristics are always hyperboliclike and could be hysteretic if the length of the microbridge satisfies the condition $u^{*}[L / \xi(T)]^{2} \gg 1$ [this result was found for a microbridge with length $L=\xi(T)$; see
Refs. 11 and 12], which roughly corresponds to our criterium $\tau_{|\Delta|} / \tau_{\delta \varphi} \sim u^{*}[L / \xi(T)]^{2} \gtrsim 1$ taken at $I=I_{c}$.

Equation (11) with arbitrary $u^{*}$ can only be used qualitatively instead of Eq. (6) for short microbridges $L<$ $\xi(T)$ where the electric field is almost constant across the microbridge. The parameter $u^{*}$ defines not only $\tau_{|\Delta|}$, but it also determines the penetration length of the electric field in the superconductor $L_{E} \sim 1 / \sqrt{u^{*}}$ (or $L_{E} \sim 1 / \sqrt{\tau_{\text {in }}}$ with the replacement $\left.u^{*} \rightarrow 2 \tau_{i n}|\Delta| / \hbar\right)$, which is rather different from $L_{E} \sim \sqrt{\tau_{\text {in }}}$ found from Eqs. (6) and (7). For the case of long microbridges $L \gtrsim \xi(T)$, it leads to drastic changes if one compares Eqs. (6) and (11): the current interval over which dynamical phase-slip processes occur decreases with increasing $u^{*}$, while it increases with increasing $\tau_{i n}{ }^{13,33}$

In a form close to Eq. (6), the time-dependent GinzburgLandau equation was used for analytical calculations of the IV characteristics of short superconducting microbridges in Ref. 26. But, the author used the wrong assumption that relaxation of the nonequilibrium occurs due to diffusion alone [see Eq. (14) in Ref. 26] and it was found to be a weak [see Eq. (12) and Fig. 5 in Ref. 34] decrease of the resistance (at $I \gtrsim I_{c}$ ) for relatively small $\tau_{i n}$. The decrease of the resistance with increasing $u^{*}$ in the framework of Eq. (11) was found for short $S-S^{\prime}-S$ (Ref. 7) and $S-N-S$ (Ref. 10) superconducting microbridges, but those authors did not study the case of large $u^{*}$ and did not observe an increase of the resistance for relatively large $u^{*}$.

In Refs. 24 and 25, the limiting case of short microbridge $L<\xi_{0}$ was studied in the framework of microscopic theory near $T_{c}$. The authors found a decrease of the resistance of the superconducting microbridge when $\tau_{i n} e V_{c} / \hbar \ll 1$ [where $V_{c}=I_{c} R_{N}$ and $\left.\hbar / e V_{c} \simeq \tau_{\delta \phi}\left(I=I_{c}\right)\right]$, but, in contrast to our results and the result of Refs. 7 and 26, the resistance in Refs. 24 and 25 did not depend on the length of the microbridge. Aside from this, the hysteresis of IV characterisitcs in Refs. 24 and 25 appeared when $\tau_{i n} e V_{c} / \hbar \sim \tau_{\text {in }} / \tau_{\delta \phi} \gg 1$, while according to our calculations, in the local limit the hysteresis appears when $\tau_{|\Delta|} / \tau_{\delta \phi} \simeq\left(L / \xi_{0}\right)^{2}\left(1-T / T_{c}\right) \tau_{i n} / \tau_{\delta \phi} \gg 1$. It is not clear how, starting from our results [which were found for microbridges with $\xi_{0}<L<\xi(T)$ ], one would continuously transit to the results found in Refs. 24 and 25 (which were found for microbridges with $L<\xi_{0}$ ) by decreasing the length of the microbridge.

\section{B. Long microbridge}

Now, we discuss the results for a long microbridge. The same idea about the competition between the two relaxation times $\tau_{|\Delta|}$ and $\tau_{\delta \phi}$ will be used in order to explain the monotonous increase of the voltage with increase of $\tau_{\text {in }}$ (at fixed current) and the hysteresis of the IV characteristics for the parameters of Fig. 2(b). In comparison with short microbridges, we should replace $L$ by $\xi(T)$ in Eq. (8) and we obtain the following estimate for $\tau_{|\Delta|} \sim \tau_{\text {in }} /\left(1-T / T_{c}\right)^{1 / 2}$, which coincides with the well-known expression for the characteristic variation of $|\Delta|$ with time at $T \sim T_{c}$ in the spatially uniform case ca,22 $^{20|\Delta|} \simeq 3.7 \tau_{\text {in }} k_{B} T_{c} / \Delta_{\text {lead }}$. To estimate $\tau_{\delta \varphi}$, we should use the depairing current $I_{c}=\sqrt{4 / 27} \pi \Delta_{\text {lead }}^{2} L /$ $4 e k_{B} T_{c} R_{N} 2 \xi(T)$ and take into account that the electric field exists over a length $L_{E} / \xi_{0} \sim \tilde{\tau}_{i n}^{1 / 2} /\left(1-T / T_{c}\right)^{1 / 419}$ near the 
phase-slip center, which could be much larger than $\xi(T) / \xi_{0} \sim$ $1 /\left(1-T / T_{c}\right)^{1 / 2}$. Therefore, we may distinguish two cases: $\xi(T)<L_{E}(T) \ll L$ and $\xi(T)<L \lesssim L_{E}(T)$.

In the first case, the voltage drop occurs over the length $2 L_{E}\left[\tau_{\delta \phi} \sim L /\left(2 L_{E} R_{N} I\right)\right]$ and, in the second case, the voltage drop occurs across the whole microbridge $\left[\tau_{\delta \phi} \sim 1 /\left(R_{N} I\right)\right]$. Therefore, we obtain

$$
\begin{array}{cc}
\frac{\tau_{|\Delta|}}{\tau_{\delta \varphi}} \simeq \tilde{\tau}_{i n}\left(1-T / T_{c}\right)^{1 / 2} \frac{I}{I_{c}} \frac{L_{E}(T)}{\xi(T)}, & L_{E}(T) \ll L \\
\frac{\tau_{|\Delta|}}{\tau_{\delta \varphi}} \simeq \tilde{\tau}_{i n}\left(1-T / T_{c}\right)^{1 / 2} \frac{I}{I_{c}} \frac{L}{\xi(T)}, & L_{E}(T) \gtrsim L .
\end{array}
$$

In both cases, the inequality $\tau_{|\Delta|} / \tau_{\delta \varphi} \gtrsim 1$ is already satisfied at $T=0.99 T_{c}$ for almost all values of $\tilde{\tau}_{\text {in }}$ presented in Fig. 2(b). It explains the monotonous dependence of the voltage for fixed current and the enhanced hysteresis in comparison with the short microbridge [see Fig. 2(a)]. As in the case of short microbridges, we may estimate the retrapping current from the condition $\tau_{|\Delta|} / \tau_{\delta \varphi}\left(I_{r}\right) \lesssim 1$ (see also Refs. 13 and 35)

$$
\begin{aligned}
I_{r} \lesssim \frac{I_{c}}{\tilde{\tau}_{i n}\left(1-T / T_{c}\right)^{1 / 2}} \frac{\xi(T)}{L_{E}(T)}, & L_{E}(T) \ll L \\
& I_{r} \lesssim \frac{I_{c}}{\tilde{\tau}_{i n}\left(1-T / T_{c}\right)^{1 / 2}} \frac{\xi(T)}{L}, \quad L_{E}(T) \gtrsim L .
\end{aligned}
$$

In both cases, the ratio $I_{r} / I_{c}$ is much smaller as compared to short microbridges for the same temperature and the same inelastic relaxation time, which is mainly connected with a larger value for $\tau_{|\Delta|}$ in long microbridges (compare $\tau_{|\Delta|}$ for short and long microbridges). Furthermore, as in the case of short microbridges, there exists a minimal voltage $V^{*}=V\left(I_{r}\right) \sim 1 / \tau_{|\Delta|}>0$ (when hysteresis exists) and the maximal period of oscillations of $|\Delta|$ is proportional to $\tau_{|\Delta|}$ and does not depend on the length of the long microbridge.

To conclude this section, we should note that, for relatively long microbridges such that $\xi(T)<L<L_{E}(T)$, the temperature dependence of $I_{r} \sim\left(1-T / T_{c}\right)^{1 / 2}$ [see Eq. (13b)] is the same as for the retrapping current in the model with Joule dissipation. ${ }^{4}$ But the validity region of this expression is restricted to temperatures near $T_{c}$ where Eqs. (6) and (7) are valid.

\section{SHORT AND LONG SUPERCONDUCTING MICROBRIDGES IN THE NONLOCAL LIMIT}

\section{A. Short microbridge}

In this section, we go beyond the validity region of the time-dependent Ginzburg-Landau equations. In our numerical calculations, in contrast with the preceding section, we use an even number of grid points in order to put the position where the order parameter goes to zero between two grid points. Further, when we refer to any value in the phase-slip center, we mean the value in the grid point next to the phase-slip center [at the distance $\delta x=\xi_{0} / 2 \ll \xi(T)$ ].

In our preceding paper, ${ }^{23}$ we found that, in the nonlocal limit, the oscillations of the order parameter in the superconducting microbridge may heat the quasiparticles on average in time. The coupling terms in Eqs. (1a) and (1b) are responsible for some kind of cooling of quasiparticles and strongly affect the IV characteristics. Here, we present a direct comparison between the results found from the nonlocal and the local limits.

In Fig. 4, we present the current voltage characteristics of a microbridge with length $L=7 \xi_{0}$ at $T=0.99 T_{c}$ for different values of the inelastic relaxation time $\tau_{\text {in }}$ found from the numerical solution of Eqs. (1)-(5). To show the influence of the coupling terms $D \nabla\left(j_{\epsilon} \delta f_{L}\right)$ and $D \nabla\left(j_{\epsilon} f_{T}\right)$ on the IV characteristics, we calculated them in the absence [Fig. 4(a)] and the presence of those terms [Fig. 4(b)].

The IV characteristics without coupling terms look qualitatively similar to those in the local limit [compare Figs. 2(a) and 4(a)]. The voltage at fixed current first decreases and then increases with increasing $\tau_{\text {in }}$ and for relatively large $\tau_{\text {in }}$ hysteresis appears. Adding the coupling terms changes the behavior at large voltages ${ }^{23}$ and leads to qualitative differences with the local limit: the voltage decreases with increasing $\tau_{\text {in }}$ [see also the IV curves in Fig. 3(b) of Ref. 23].

Let us first discuss the main differences between the local and nonlocal limits at low voltages where the effect of the coupling terms is relatively weak and their presence does not lead to qualitative differences in the IV characteristics [compare Figs. 4(a) and 4(b) at $I \lesssim 1.3 I_{c}$ ]. In Fig. 5, we present the dependence of $|\Delta|, I_{s}$, and $\Phi_{1}$ on the phase

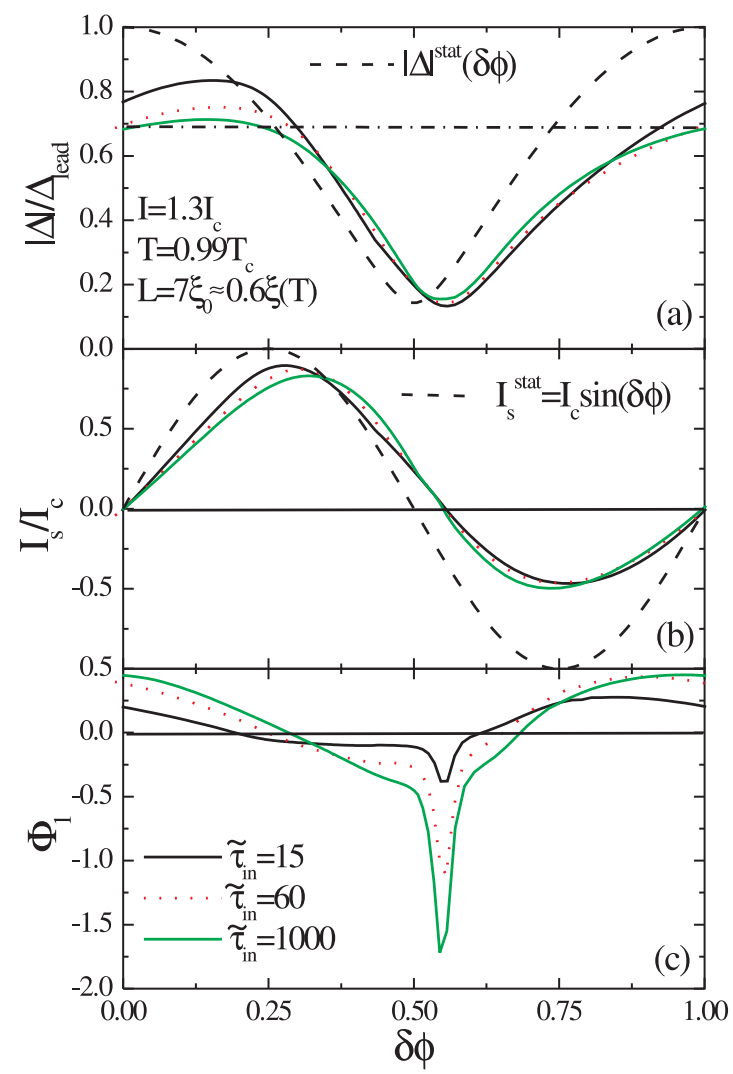

FIG. 5. (Color online) Dependence of the magnitude of the order parameter, the superconducting current, and the potential $\Phi_{1}$ in the center of the short microbridge $\left[L=7 \xi_{0} \sim 0.6 \xi(T)\right.$ at $\left.T=0.99 T_{c}\right]$ on the phase difference across the sample at $I=1.3 I_{c}$ and different inelastic relaxation times $\tilde{\tau}_{\text {in }}$ found in the nonlocal limit [with presence of coupling terms in Eqs. (1a) and (1b)]. The black dashed line in (a) shows the minimal value of $|\Delta|$ in the stationary state when $\delta \phi=\pi / 2$. 


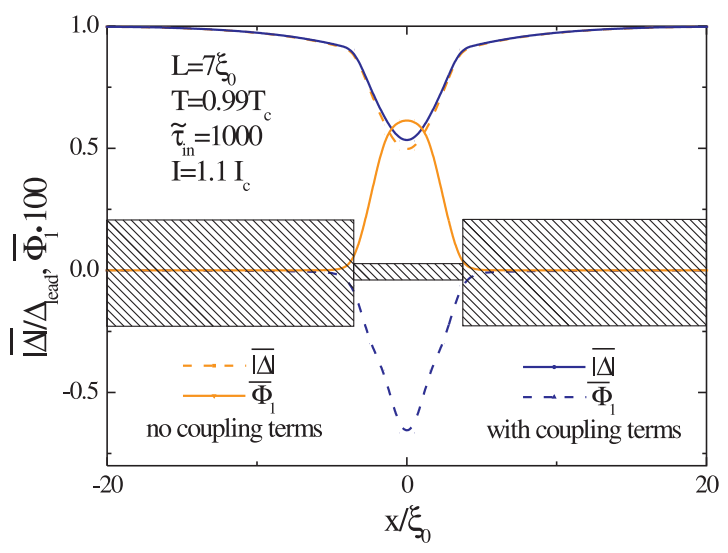

FIG. 6. (Color online) Distribution of the time-averaged potential $\bar{\Phi}_{1}$ and the order parameter $\overline{|\Delta|}$ in the short microbridge calculated with and without coupling terms in Eqs. (1a) and (1b). The shaded area represents a sketch of the microbridge with leads.

difference across the microbridge found from a numerical solution of Eqs. (1)-(5) with coupling terms at $I=1.3 I_{c}$. We see that the amplitude in the variations of $|\Delta|$ is larger in the nonlocal limit than in the local one and the dependence $I_{S}(\delta \phi)$ is close to a sinusoidal one (compare Figs. 3 and 5). The second difference is in the sign of the potential $\Phi_{1}$. In the local limit, the sign of $\Phi_{1}$ is determined by the sign of the time derivative of $|\Delta|$, i.e., $\Phi_{1} \sim \partial|\Delta| / \partial t .^{17,18}$ In the nonlocal limit, the sign of $\Phi_{1}$ may not coincide with the sign of $\partial|\Delta| / \partial t$ when $|\Delta|$ varies faster in time than $f(\epsilon)$. It leads to a faster initial increase of the order parameter after the phase-slip event than in the local limit ${ }^{36}$ and to positive $\Phi_{1}$ during a relatively large time interval after the phase-slip event even when $\partial|\Delta| / \partial t \lesssim 0$ [see Fig. 5(c)].

Positive (negative) $\Phi_{1}$ means, in some respect, heating (cooling) of quasiparticles because, from the structure of Eq. (3) and the definition of $\Phi_{1}$, it follows that the effect of the nonequilibrium $f_{L}(\epsilon)$ may be interpreted integrally in terms of the effective temperature of the quasiparticles: $T_{\text {eff }}=T+T_{c} \Phi_{1}$. Within the local limit, the time-averaged $\Phi_{1}=0$, while in the nonlocal case, $\bar{\Phi}_{1}$ can be positive [when one neglects coupling terms in Eqs. (1a) and (1b)] and negative (when one takes these terms into account) (see Fig. 6).

Despite the above differences, hysteresis appears in both local and nonlocal limits at large $\tilde{\tau}_{i n}$ and we argue that the main reason for this is the large growth time of $|\Delta|$ in comparison with the growth time of $\delta \phi$. Indeed, nonlocal effects speed up the initial stage of the increase of $|\Delta|$ after the phase-slip event, but when $|\Delta|$ becomes large enough, it varies in time as in the case of the local limit: ${ }^{36}$ the larger $\tilde{\tau}_{i n}$, the slower changes $|\Delta|$ [see also Fig. 5(a), which shows the time dependence of $|\Delta|$ because the phase difference changes in time]. Due to nonlocal effects, $\Phi_{1}$ stays positive and large even when $|\Delta|$ reaches its maximal value [in contrast with the local limit ; see Fig. 5(c)], which means a local in time heating of quasiparticles. As a result, even when there is a time-averaged cooling of quasiparticles (see Fig. 6), hysteresis still persists [see Fig. 4(b)]. In the absence of coupling terms in Eqs. (1a) and (1b), the potential $\Phi_{1}$ is positive during almost the whole period of oscillations and hysteresis is larger

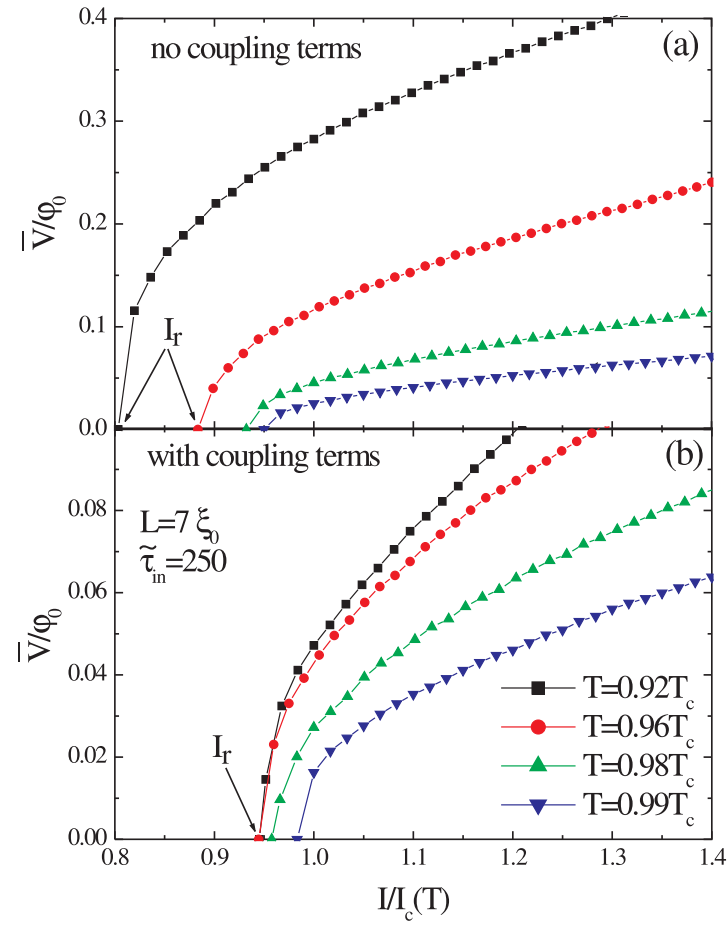

FIG. 7. (Color online) Current voltage characteristics of a short superconducting microbridge with length $L=7 \xi_{0}$ and $\tilde{\tau}_{\text {in }}=250$ calculated at different temperatures without (a) and with (b) coupling terms in Eqs. (1a) and (1b).

than the one found when taking into account the coupling terms.

In Figs. 7(a) and 7(b), we show the IV characteristics of a short microbridge at different temperatures calculated for one value of $\tau_{i n}$. At low temperature, the hysteresis becomes more pronounced; the same effect exists in the local limit too and it is connected with an increase of the difference between $\tau_{|\Delta|}$ and $\tau_{\delta \phi}$. The presence of coupling terms in Eqs. (1a) and (1b) makes the hysteresis weaker because they provide cooling of quasiparticles and only the large $\tau_{|\Delta|}$ is still responsible for hysteresis.

\section{B. Long microbridge}

In Figs. 8 and 9, we present IV characteristics of a long microbridge $L=21 \xi_{0}$ calculated at $T=0.99 T_{c}$ (Fig. 8) and $T=0.96 T_{c}$ (Fig. 9) for different values of $\tau_{i n}$ in the absence and the presence of coupling terms in Eqs. (1a) and (1b). First of all, we should note that the effect of coupling terms is similar to the one for short microbridges and becomes pronounced already at $T=0.99 T_{c}$. Their presence leads to an effective cooling of the quasiparticles and to a decrease of the voltage with increasing $\tau_{i n}$ for fixed value of the current $I \gtrsim I_{c}$.

Second, the hysteresis considerably increases in comparison with the one found for a short microbridge when taken at the same temperature and with the same $\tilde{\tau}_{i n}$ (compare Figs. 4 and 8 and Fig. 3 from Ref. 23 with Fig. 9). The same effect appears already within the framework of the 


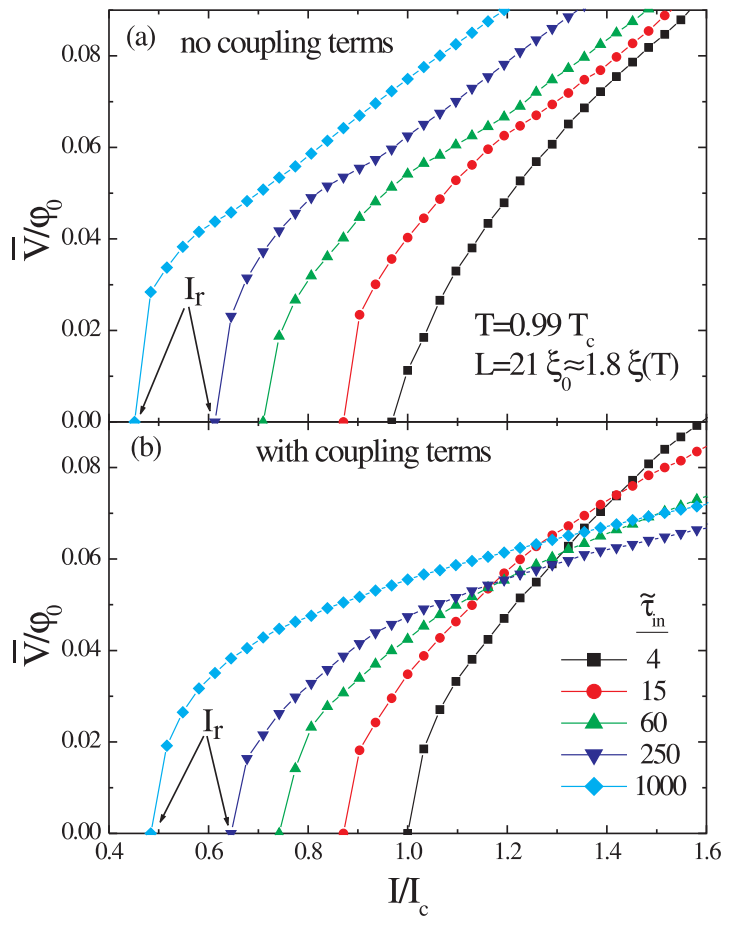

FIG. 8. (Color online) Current voltage characteristics of a long superconducting microbridge with length $L=21 \xi_{0}$ calculated for different $\tau_{\text {in }}$ at $T=0.99 T_{c}$ without (a) and with (b) the coupling terms in Eqs. (1a) and (1b).

time-dependent Ginzburg-Landau equations (6) and (7) [see Figs. 2(a) and 2(b)]. In the local limit, the increase of the hysteresis (decreasing the retrapping current $I_{r}$ ) is connected with an increase of the time relaxation of $|\Delta|$, which is inversely proportional to $L^{2}$ for short microbridges and $\xi(T)^{2}$ for long microbridges. In the nonlocal case, the same reason is mainly responsible for the increase of the hysteresis because the presence or the absence of coupling terms (and therefore time-averaged cooling and heating of quasiparticles) does not affect the retrapping current $I_{r}$ considerably [compare Figs. 8(a), 8(b) and 9(a), 9(b)]. Note that, in contrast to the short microbridge, $\overline{\Phi_{1}}$ is positive in the phase-slip core both in the absence and the presence of the coupling terms (see Fig. 10). The reason is that the phase-slip core is larger in the case of a long microbridge than in the short one (compare Figs. 6 and 10), and sources of cooling [term $D \nabla\left(j_{\epsilon} f_{T}\right)$ in Eq. (1a)] and heating (oscillations of $|\Delta|$ ) are separated in space.

The coupling term provides an enhancement of the order parameter on the length scale $\sim L_{i n}>\xi(T)$ (where an electric field is present) near the phase-slip center (see Fig. 10). But, this result should be considered carefully because the deviation from equilibrium $\delta f_{L}$ may become comparable with $f_{L}^{0}$ at $\epsilon \simeq \Delta_{\text {lead }}$ [see Fig. 11(b) ] and the relaxation-time approach for the electron-phonon collision integral in Eqs. (1a) and (1b) becomes invalid. We found that the condition $\overline{\delta f_{L}} / f_{L}^{0} \ll 1$ is fulfilled in case of no coupling terms at all considered currents I and $\tilde{\tau}_{i n}$ [for illustration, see Fig. 11(a)] and in the case with coupling terms for large $\tau_{i n}$ only near the retrapping current $I \sim I_{r}$ [see Fig. 11(b)].

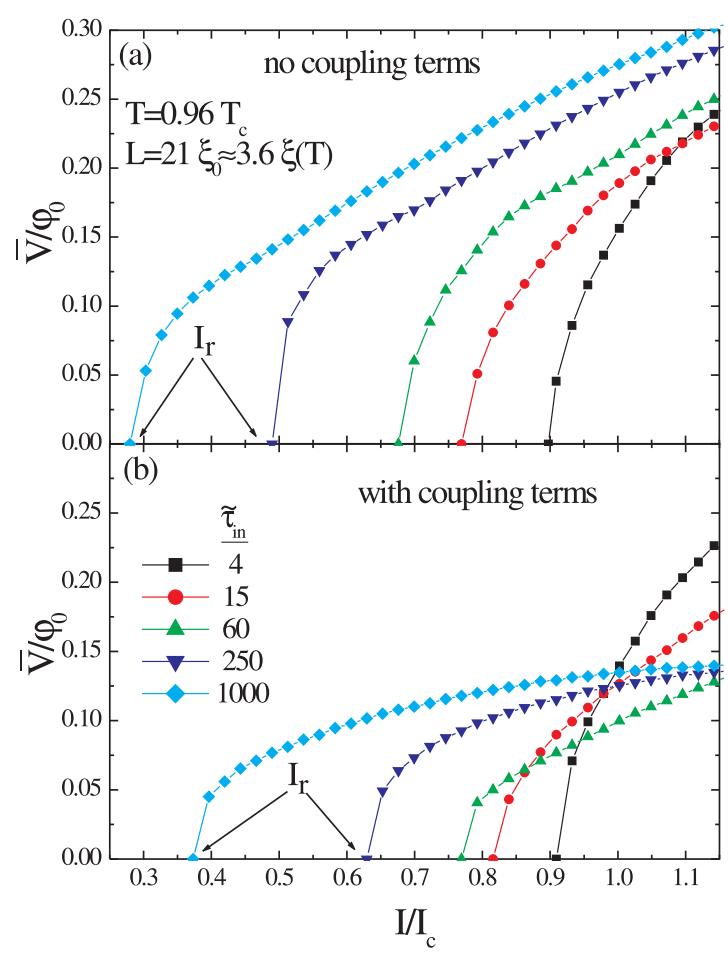

FIG. 9. (Color online) Current voltage characteristics of a long superconducting microbridge with length $L=21 \xi_{0}$ calculated for different $\tau_{i n}$ at $T=0.96 T_{c}$ without (a) and with (b) the coupling terms in Eqs. (1a) and (1b).

\section{DISCUSSION}

In the derivation of Eqs. (1)-(5), it was assumed that the inelastic relaxation time due to the electron-electron interaction is much larger than the inelastic relaxation time due to electron-phonon interaction. But, in many low-temperature superconductors (for example, in aluminum and zinc) already at $T \sim T_{c}$, these relaxation times are of the same order and one can expect a strong influence of the electron-electron interaction on the energy profile of $\delta f_{L}$. From a physical point of view, the electron-electron interaction thermalizes $f(\epsilon)$ on the time scale $\tau_{e-e}$. Therefore, we may expect that the large peak near $\epsilon \simeq \Delta_{\text {lead }}$ will be smeared out not only by taking into account the correct expression for the electron-phonon collision integral (when $\delta f_{L} \sim f_{L}^{0}$ ), but also by electronelectron interaction. The cooling effect will be weakened and the shape of the IV curves will be between the curves presented in Figs. 8(a) and 8(b) and 9(a) and 9(b).

Also, one more simplification was made in connection with the phonon subsystem: it was supposed that the phonons are in equilibrium. This assumption should be fulfilled for variable thickness bridges, when the narrow and thin microbridge is connected with wide and thick banks and one may assume that nonequilibrium phonons escape fast to the leads (contrary to the electrons with energy $\epsilon<\Delta_{\text {lead }}$ for which an energy barrier exists). In the case of the Dayem microbridge (in which the thickness of the microbridge is the same as the thickness of the leads), the nonequilibrium phonons can not effectively run away from the microbridge and one should take into account their heating by Joule dissipation. 


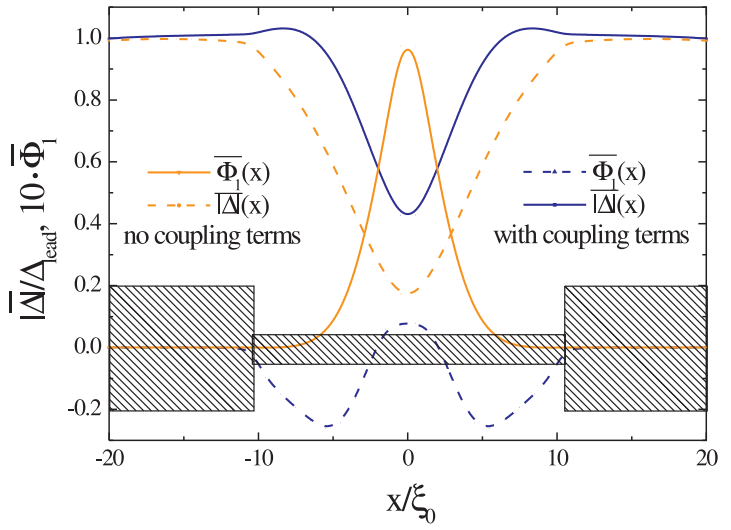

FIG. 10. (Color online) Distribution of the time-averaged potential $\Phi_{1}$ and the order parameter $|\Delta|$ in the long microbridge $\left(L=21 \xi_{0}, T=0.96 T_{c}, \bar{\tau}_{i n}=250, I=1.2 I_{c}\right)$ calculated with and without coupling terms in Eqs. (1a) and (1b). The shaded area is a sketch of the microbridge with leads.

In this connection, we also would like to discuss another effect of the geometry of the microbridge [which is especially important for short microbridges $L \lesssim \xi(T)$ ] on the nonequilibrium electrons. In variable thickness microbridges, the regions next to the microbridge are almost not influenced by a large supercurrent and one may assume that $\Delta_{\text {lead }}=$ $\Delta_{\mathrm{GL}}\left(1-T / T_{c}\right)^{1 / 2}$. In the Dayem microbridge, the order parameter will be suppressed in the area with size about the width of the microbridge by the large superconducting current $I \gtrsim I_{c} .{ }^{1}$ It will affect the nonequilibrium distribution function $\delta \tilde{f_{L}}$ in the microbridge because, in this case, the relaxation due

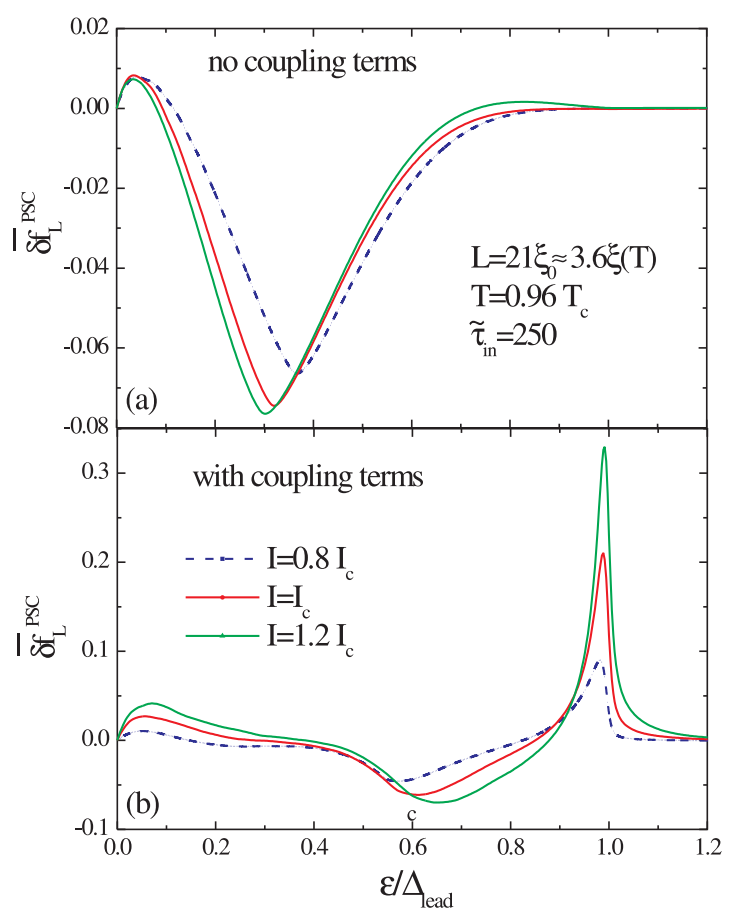

FIG. 11. (Color online) Energy dependence of the time-averaged $\delta f_{L}$ in the center of a long microbridge (in the phase-slip center) at different currents in the absence (a) and the presence (b) of coupling terms in Eqs. (1a) and (1b).

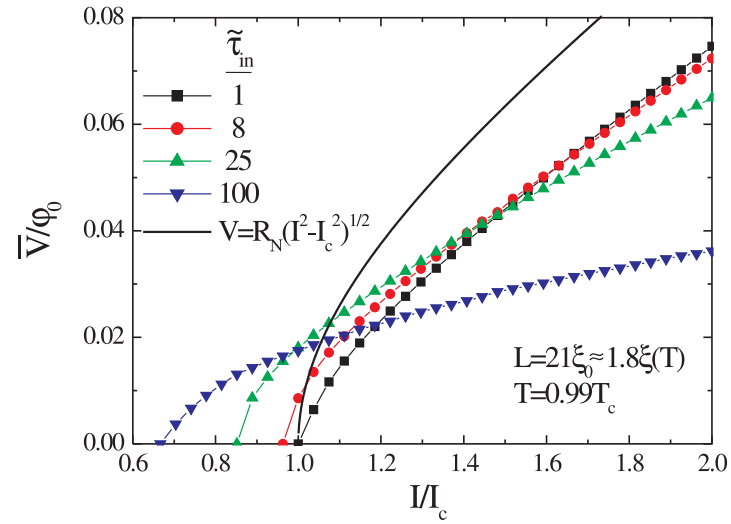

FIG. 12. (Color online) Current voltage characteristics of a long superconducting microbridge calculated in the regime of decreasing current on the basis of a numerical solution of the time-dependent Ginzburg-Landau equations with additional cooling term.

to diffusion will be larger for quasiparticles with $\epsilon \lesssim \Delta_{\text {lead }}$. Therefore, the effect of the coupling terms should be weakened (they give the main contribution for $\epsilon \sim \Delta_{\text {lead }}$ ) and the cooling effect will be smaller in the Dayem microbridge in comparison with the variable thickness microbridge.

An important effect may also come from the finite width of the microbridge. In our calculations, we assumed that the width of the microbridge is much smaller than its length, which provides uniform current distribution over the width of the microbridge. In the case when $W \gtrsim L$, current distribution becomes nonuniform (see Ref. 37) and it may considerably influence the dynamics of the order parameter in the microbridge (see discussion in Ref. 23).

We should note that cooling of quasiparticles can be taken into account in the local limit. The contribution to the cooling comes from the term $D \nabla\left(j_{\epsilon} f_{T}\right) \simeq 2 D N_{2} R_{2}(Q \cdot E) \partial f_{L}^{0} / \partial \epsilon$ (Ref. 23) in the kinetic equation [Eq. (1a)]. In the local limit, it leads to the appearance of an additional term in the generalized time-dependent Ginzburg-Landau equation

$$
\begin{aligned}
& \frac{\pi \hbar}{8 k_{B} T_{c}} \frac{1}{\sqrt{1+(\Gamma|\Delta|)^{2}}}\left(\frac{\partial}{\partial t}-\frac{i 2 e \varphi}{\hbar}+\frac{\Gamma^{2}}{2} \frac{\partial|\Delta|^{2}}{\partial t}\right) \Delta \\
& \quad=\xi_{\mathrm{GL}}^{2} \frac{\partial^{2} \Delta}{\partial x^{2}}+\left(1-\frac{T}{T_{c}}-\frac{|\Delta|^{2}}{\Delta_{\mathrm{GL}}^{2}}+\xi_{\mathrm{GL}}^{2} \mathbf{e} \Gamma(\mathbf{Q} \cdot \mathbf{E})\right) \Delta,
\end{aligned}
$$

where we used the same local approximation limit for $N_{1}, N_{2}$, and $R_{2}$ as in Refs. 17 and 18. In Fig. 12, we present the IV characteristics for the parameters of Fig. 2(b) calculated using Eq. (14) and one can see qualitative agreement between these results and results presented in Fig. 8(b) [compare also with Fig. 2(b)].

\section{CONCLUSION}

The reason for the hysteresis of the IV characteristics of short and long variable thickness microbridges near the critical temperature are similar for both the local $\left[L_{i n} \ll \xi(T)\right]$ and the nonlocal $\left[L_{i n} \gg \xi(T)\right]$ limits. The hysteresis is connected with the large relaxation time of $|\Delta|$ in the phase-slip core in 
comparison with the time scale for the variation of $\delta \phi$ across the short microbridge or across the phase-slip core (in case of long microbridge).

In short and long microbridges, $\tau_{|\Delta|}$ increases with increasing inelastic relaxation time $\tau_{\text {in }}$ (in the local limit $\tau_{|\Delta|} \sim \tau_{\text {in }}$ ). Note that $\tau_{|\Delta|}$ depends on the length of the short microbridge (in the local limit $\tau_{|\Delta|} \sim 1 / L^{2}$ ) and on temperature. In contrast, the time variation of $\delta \phi$ depends mainly on the temperature because it is inversely proportional to the voltage across the microbridge [and $V \sim R_{N} I_{c}(T)$ ] and $\tau_{\delta \phi}$ decreases with decreasing temperature. At some temperature below the critical one, $|\Delta|$ may vary much slower in time than $\delta \phi$ and it provides the condition for the existence of the dynamical phase-slip process at $I_{r}<I<I_{c}$ resulting in hysteresis of the IV characteristics.

In the nonlocal limit, the difference between $\tau_{|\Delta|}$ and $\tau_{\delta \phi}$ leads to a time-averaged heating of the quasiparticles in the phase-slip core and, hence, to an additional source of hysteresis. Due to the coupling of the longitudinal and transverse parts of the quasiparticle distribution function, there is an effective cooling of the quasiparticles in the area outside of the phase-slip core where an electric field exists and oscillations of $|\Delta|$ have small amplitude. In short microbridges, this cooling partially compensates the heating of quasiparticles in the phase-slip core and the hysteresis is smaller than predicted by the local model. In long microbridges, the cooled regions are far from the phase-slip core and cooling itself is weaker at low voltages and it slightly affects the retrapping current $I_{r}$. At high voltages, the cooling for both short and long microbridges becomes stronger and it results in a decrease of the voltage at fixed current with increasing $\tilde{\tau}_{i n}$. The last effect is also found in the local limit both for long and short microbridges if one takes into account the additional cooling term [see Eq. (14)] in the time-dependent Ginzburg-Landau equation. Note that the voltage decrease partially exists in short microbridges at low $\tau_{\text {in }}$ even without the cooling term in the TDGL equation (in this case, the voltage decrease is connected with the increase of $\tau_{|\Delta|} \sim \tilde{\tau}_{\text {in }}$, but not with the time-averaged cooling).

The good candidates for observation of the predicted effects are low-temperature superconductors $\mathrm{NbGe} / \mathrm{MoGe}$, TiN, and Sn for which $\tau_{e-p h}$ is shorter than $\tau_{e-e}$ in the wide temperature interval below $T_{c}$. The length of the microbridges should be less inelastic relaxation length (to have single phase-slip center in the microbridge), and in these materials, $L_{i n}$ is about $80 \mathrm{~nm}$ for $\mathrm{NbGe} / \mathrm{MOGe},{ }^{38,39} 250 \mathrm{~nm}$ for TiN, ${ }^{40}$ and $400-500 \mathrm{~nm}$ for Sn. ${ }^{41,42}$

\section{ACKNOWLEDGMENTS}

This work was supported by the Russian Foundation for Basic Research, Russian Agency of Education under the Federal Target Programme"Scientific and educational personnel of innovative Russia in 2009-2013," the Flemish Science Foundation (FWO-Vl), and the Belgian Science Policy (IAP). *vodolazov@ipm.sci-nnov.ru

${ }^{1}$ K. K. Likharev, Rev. Mod. Phys. 51, 101 (1979).

${ }^{2} \mathrm{R}$. Tidecks, Current-Induced Nonequilibrium Phenomena in QuasiOne-Dimensional Superconductors (Springer, Berlin, 1990).

${ }^{3}$ V. N. Gubankov, K. K. Likharev, and N. M. Margolin, Fiz. Tverd. Tela 14, 935 [Sov. Phys. Solid State 14, 819 (1972)].

${ }^{4}$ W. J. Skocpol, M. R. Beasley, and M. Tinkham, J. Appl. Phys. 45, 4054 (1974).

${ }^{5}$ M. Tinkham, J. U. Free, C. N. Lau, and N. Markovic, Phys. Rev. B 68, 134515 (2003).

${ }^{6}$ D. Hazra, L. M. A. Pascal, H. Courtois, and A. K. Gupta, Phys. Rev. B 82, 184530 (2010).

${ }^{7}$ K. K. Likharev and L. A. Yakobson, Zh. Eksp. Teor. Fiz. 68, 1150 (1975) [Sov. Phys.- JETP 41, 570 (1976)].

${ }^{8}$ Y. Song, J. Appl. Phys. 47, 2651 (1976).

${ }^{9}$ H. Hojgaard-Jensen and P. E. Lindelof, J. Low Temp. Phys. 23, 469 (1976).

${ }^{10}$ R. B. van Dover, A. de Lozanne, and M. R. Beasley, J. Appl. Phys. 52, 7327 (1981).

${ }^{11}$ A. Baratoff and L. Kramer, in Proceedings of the International Conference on Superconducting Quantum Interference Devices and Their Applications (Walter de Gruyter, Berlin, 1977), pp. 51-62.

${ }^{12}$ A. L. De Lozanne and M. R. Beasley, in Nonequilibrium Superconductivity, edited by D. N. Langenberg and A. I. Larkin (Elsevier, Amsterdam, 1986), Chap. 3.

${ }^{13}$ S. Michotte, S. Matefi-Tempfli, L. Piraux, D. Y. Vodolazov, and F. M. Peeters, Phys. Rev. B 69, 094512 (2004).
${ }^{14}$ F. Giazotto, T. T. Heikkilä, A. Luukanen, A. M. Savin, and J. P. Pekola, Rev. Mod. Phys. 78, 217 (2006).

${ }^{15}$ A. Schmid and G. Schön, J. Low Temp. Phys. 20, 207 (1975).

${ }^{16}$ A. I. Larkin and Yu. N. Ovchinnikov, Zh. Eksp. Teor. Fiz. 73, 299 (1977) [Sov. Phys. JETP 73, 155 (1977)].

${ }^{17}$ L. Kramer and R. J. Watts-Tobin, Phys. Rev. Lett. 40, 1041 (1978).

${ }^{18}$ R. J. Watts-Tobin, Y. Krähenbühl, and L. Kramer, J. Low Temp. Phys. 42, 459 (1981).

${ }^{19}$ W. J. Skocpol, M. R. Beasley, and M. Tinkham, J. Low Temp. Phys. 16, 145 (1974)

${ }^{20}$ M. Tinkham, Introduction to Superconductivity (McGraw-Hill, New York, 1996).

${ }^{21}$ L. G. Aslamazov and A. I. Larkin, Zh. Eksp. Teor. Fiz. 70, 1340 (1976) [Sov. Phys. JETP 43, 698 (1976)].

${ }^{22}$ A. Schmid, G. Schön, and M. Tinkham, Phys. Rev. B 21, 5076 (1980).

${ }^{23}$ D. Y. Vodolazov and F. M. Peeters, Phys. Rev. B 83, 224523 (2011) (see also e-print arXiv:1105.5489).

${ }^{24}$ S. N. Artemenko, A. F. Volkov, and A. V. Zaitsev, Zh. Eksp. Teor. Fiz. 76, 1816 (1979) [Sov. Phys. JETP 49, 924 (1979)].

${ }^{25}$ A. V. Zaitsev, Zh. Eksp. Teor. Fiz. 78, 221 (1980) [Sov. Phys. JETP 51, 111 (1980)]; 79, 2015 (1980) [52, 1018 (1980)].

${ }^{26}$ A. A. Golub, Zh. Eksp. Teor. Fiz. 71, 341 (1976) [Sov. Phys. JETP 44, 178 (1976)].

${ }^{27}$ R. S. Keizer, M. G. Flokstra, J. Aarts, and T. M. Klapwijk, Phys. Rev. Lett. 96, 147002 (2006) 
${ }^{28}$ D. Yu. Vodolazov and F. M. Peeters, Phys. Rev. B 75, 104515 (2007).

${ }^{29}$ L. G. Aslamazov and A. I. Larkin, Zh. Eksp. Teor. Fiz. Pisma Red. 9, 150 (1969) [Sov. Phys. JETP Lett. 9, 87 (1969)].

${ }^{30}$ J. A. Pals and J. Wolter, Phys. Lett. A 70, 150 (1979) (see also Ref. 20, p. 414).

${ }^{31}$ M. Tinkham, in Non-Equilibrium Superconductivity, Phonons and Kapitza Boundaries, edited by K. E. Gray (Plenum, New York, 1981).

${ }^{32}$ A. Geier and G. Schön, J. Low Temp. Phys. 46, 151 (1982).

${ }^{33}$ L. Kramer and R. Rangel, J. Low. Temp. Phys. 57, 391 (1984).

${ }^{34}$ M. Octavio, W. J. Skocpol, and M. Tinkham, Phys. Rev. B 17, 159 (1978).
${ }^{35}$ D. Y. Vodolazov, Phys. Rev. B 75, 184517 (2007).

${ }^{36}$ D. Yu. Vodolazov and F. M. Peeters, Phys. Rev. B 81, 184521 (2010).

${ }^{37}$ L. G. Aslamazov and A. I. Larkin, Zh. Eksp. Teor. Fiz. 68, 766 (1975) [Sov. Phys. JETP 41, 381 (1975)].

${ }^{38}$ D. Babic, J. Bentner, C. Surgers, and C. Strunk, Phys. Rev. B 69, 092510 (2004).

${ }^{39}$ M. Liang and M. N. Kunchur, Phys. Rev. B 82, 144517 (2010).

${ }^{40}$ F. Lefloch, C. Hoffmann, and O. Demolliens, Phys. C (Amsterdam) 319, 258 (1999).

${ }^{41}$ L. E. Musienko, I. M. Dmitrenko, and V. G. Volotskaya, Zh. Eksp. Teor. Fiz. Pis'ma Red. 31, 603 (1980) [JETP Lett. 31, 567 (1980)].

${ }^{42}$ W. Klein, R. P. Huebener, S. Gauss, and J. Parisi, J. Low Temp. Phys. 61, 413 (1985). 